\title{
A New Look at Neon-C and SEP-Neon
}

\author{
R. A. Mewaldt ${ }^{*}$, R. C. Ogliore* , G. Gloeckler ${ }^{+}$and G. M. Mason ${ }^{+}$ \\ *California Institute of Technology, Pasadena, CA 91125 USA \\ ${ }^{+}$University of Maryland, College Park, MD 20742 USA
}

\begin{abstract}
Studies of the isotopic composition of neon in lunar soils, meteorites, and interplanetary dust particles have revealed several distinct components. In addition to implanted solar wind, which has a ${ }^{20} \mathrm{Ne} /{ }^{22} \mathrm{Ne}$-abundance ratio of 13.7, there is an additional component with ${ }^{20} \mathrm{Ne} /{ }^{22} \mathrm{Ne} \approx 11.2$, originally attributed to higher-energy solar energetic particles. Using data from the Advanced Composition Explorer, we have measured the fluence of solar wind, suprathermal particles, solar energetic particles and cosmic rays from $\sim 0.3 \mathrm{keV} /$ nucleon to $\sim 300 \mathrm{MeV} /$ nucleon over an extended time period. We use these measured spectra to simulate the present-day depth distribution of $\mathrm{Ne}$ isotopes implanted in the lunar soil. We find that the suprathermal tail of the solar wind, extending from a few $\mathrm{keV} /$ nucleon to several $\mathrm{MeV} /$ nucleon with a power law spectrum, can produce ${ }^{20} \mathrm{Ne} /{ }^{22} \mathrm{Ne}$ abundance ratios in the lunar soil that are similar to the measured composition, although there remain significant questions about the extent to which the present-day intensity of suprathermal ions is sufficient to explain the lunar observations.
\end{abstract}

\section{INTRODUCTION}

Solar-wind noble gases implanted in the lunar soil preserve a record of solar wind activity over the history of the solar system. Beginning with the return of the first Apollo samples from the moon, this record has revealed evidence for several distinct isotopic components implanted in lunar materials (e.g., Pepin et al. [1]). In addition to implanted solar wind and at least two primordial components, there is evidence for a component that contains excesses of the heavy isotopes of $\mathrm{He}, \mathrm{Ne}, \mathrm{Ar}, \mathrm{Kr}$, and $\mathrm{Xe}$ when compared to the composition of the present-day solar wind (see review by Wieler [2]). There is also evidence for an additional component of nitrogen, depleted in ${ }^{15} \mathrm{~N}$ that may be related [3]. The best measurements of implanted noble gases have been obtained by step-wise etching of small grains of lunar and meteoritic material [2]. This process gradually releases gas from deeper and deeper sites that is then analyzed by mass spectroscopy techniques. A complication is that the depth from which gas is released during a given step is not well defined.

The noble gas components enriched in heavy isotopes were at first labeled He-C, Neon-C, etc [4]. They were later ascribed to solar energetic particles [5, 6], because they are implanted somewhat deeper in lunar samples than solar wind, and identified as the "SEP" component. However, it appears that the amount of implanted gas associated with this component is $\sim 10 \%$ to $40 \%$ of the solar wind $[7,2]$, orders of magnitude greater than expected from the present-day frequency and size of solar-particle events associated with flares and coronal mass ejections.
A number of possible origins for the SEP component have been considered (see e.g., [2, 8, 9, 10]). Recently, Wimmer-Schweingruber and Bochsler $[11,12]$ suggested that this component was implanted as the Sun passed through dense interstellar clouds during its journey through the Galaxy. In their scenario the SEP component represents a sample of interstellar matter, the isotopic anomalies result from galactic evolution effects since the birth of the solar system, and the lunar soil provides a "travel diary" of the voyage of the solar system through the Galaxy.

In this paper we consider a solar/heliospheric origin for the SEP component. Using data from several instruments on the Advanced Composition Explorer (ACE), Mewaldt et al. [13] have measured the presentday fluences of energetic $\mathrm{He}, \mathrm{O}$, and $\mathrm{Fe}$ from $\sim 0.3$ $\mathrm{keV} /$ nucleon to $\sim 300 \mathrm{MeV} /$ nucleon, ranging from solar wind to cosmic ray energies. This period included highspeed streams, impulsive and gradual solar particle events, events associated with corotating interaction regions (CIRs) as well as solar-minimum and solarmaximum fluxes of anomalous and galactic cosmic rays. In the "suprathermal" energy range from $\sim 10$ $\mathrm{keV} /$ nucleon to $\sim 10 \mathrm{MeV} /$ nucleon, all species were found to have the same power-law spectral shape [13]. Using measured energy spectra of solar wind and suprathermal particles, we have simulated the depth distribution of $\mathrm{Ne}$ isotopes implanted in lunar soil. We compare these simulations with lunar sample data in an effort to shed light on the origin of the mysterious SEP component. In this discussion we will use the term SEP to refer to this unknown component without assuming that it is due to solar energetic particles.

CP598, Solar and Galactic Composition, edited by R. F. Wimmer-Schweingruber

(C) 2001 American Institute of Physics 0-7354-0042-3/01/\$18.00 


\section{FLUENCE MEASUREMENTS}

The fluence measurements that are the basis for our simulations were obtained from four instruments on ACE during a 33-month period that starts with solarminimum conditions in 1997 and ends with solarmaximum conditions in 2000 [13]. Energetic particle fluences above $40 \mathrm{keV} /$ nucleon were integrated over the period from 1997:280 to 2000:184. The solar wind data were summed over an 11-month period from 1/99 to $11 / 99$, and then multiplied by a factor of 3 to correspond to the 33-month period covered by the higher-energy instruments. Because solar wind is less variable than higher-energy solar/interplanetary particles, the 1999 period should provide a reasonable representation of the longer period.

The spectra in Fig. 1 have a common shape. The peak at $\sim 0.8 \mathrm{keV} /$ nucleon corresponds to the slow-speed solar wind with $\sim 400 \mathrm{~km} / \mathrm{sec}$ velocity. The contribution of occasional higher-speed streams reaching $\sim 1000$ $\mathrm{km} / \mathrm{sec}$ is also apparent. Based on these spectra, it appears that the solar wind distribution extends to $\sim 8$ $\mathrm{keV} /$ nucleon, at which point there is a change in slope. Beyond this, a long, suprathermal tail extends with a power-law slope of -2 out to several MeV/nucleon. Near $\sim 10 \mathrm{MeV} /$ nucleon all three spectra exhibit a gradual "knee" and briefly steepen. Above $\sim 50$ $\mathrm{MeV} /$ nucleon, galactic cosmic rays (GCRs) make the dominant contribution. In the integral energy spectrum for oxygen in Figure 2 it is clear that the integrated fluence above $\sim 7 \mathrm{keV} /$ nucleon amounts to only a small fraction $\left(\sim 3 \times 10^{-5}\right)$ of the solar-wind fluence.

In the energy range above $\sim 10 \mathrm{keV} /$ nucleon, measurements with SWICS/ACE show that suprathermal tails on the solar wind are continuously present, even in the absence of interplanetary shocks [14]. Although, the origin of these tails is presently a subject of investigation $([14,15])$, they do not appear to be due to large, individual solar particle events such as the July 14, 2000 event, to impulsive solar flares, or to CIR events [13]. A more or less continuous process appears to be the source of most of these particles.

Three-year fluence spectra of eight elements and ${ }^{3} \mathrm{He}$ from the ULEIS and SIS instruments on ACE all show the same $\mathrm{E}^{-2}$ power-law shape from $\sim 0.04 \mathrm{MeV} /$ nucleon to $\sim 10 \mathrm{MeV} /$ nucleon [13], suggesting that this behavior is characteristic of this extended suprathermal energy range. Studies of individual solar-particle events with $\sim 1$ and $>10 \mathrm{MeV} /$ nucleon indicate that the solar particle ${ }^{20} \mathrm{Ne} /{ }^{22} \mathrm{Ne}$ ratio is, on average, somewhat less than the solar wind value $([16,17,18,19])$. However, measurements of this ratio for the 3-year fluence period are not yet available.

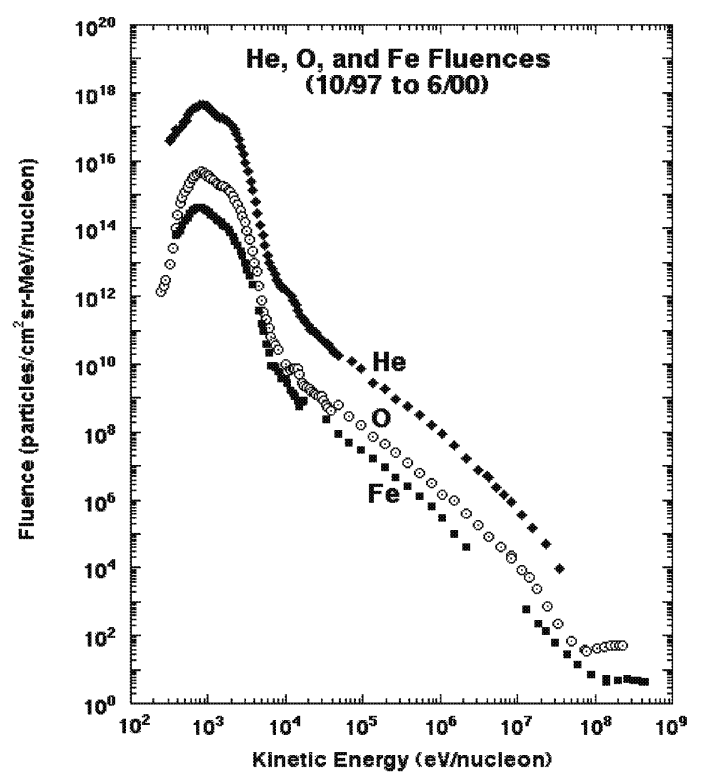

Figure 1: Fluences of $\mathrm{He}, \mathrm{O}$, and Fe nuclei measured during the period from $9 / 1997$ to $6 / 2000$ by the SWICS, ULEIS, SIS, and CRIS instruments on ACE. For additional information see Mewaldt et al. [13].

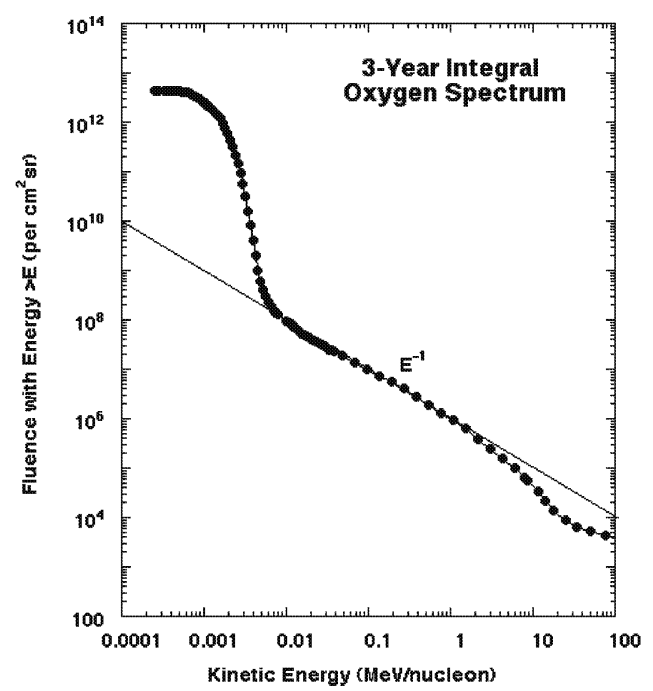

Figure 2: Integral spectrum of oxygen nuclei corresponding to the fluence spectrum in Figure 1.

\section{SIMULATED DEPTH DISTRIBUTIONS}

In order to simulate the depth distributions of energetic ions in the lunar soil we used the TRIM program [20], which performs 3-D Monte-Carlo calculations of individual ion trajectories in a variety of materials. By repeating the calculation, a distribution of penetration depths is obtained. Figure 3 shows the mean 
penetration depth of ${ }^{22} \mathrm{Ne}$ and ${ }^{20} \mathrm{Ne}$ as a function of incident kinetic energy. At a given energy/nucleon, ${ }^{22} \mathrm{Ne}$ has $\sim 10 \%$ greater range than ${ }^{20} \mathrm{Ne}$.

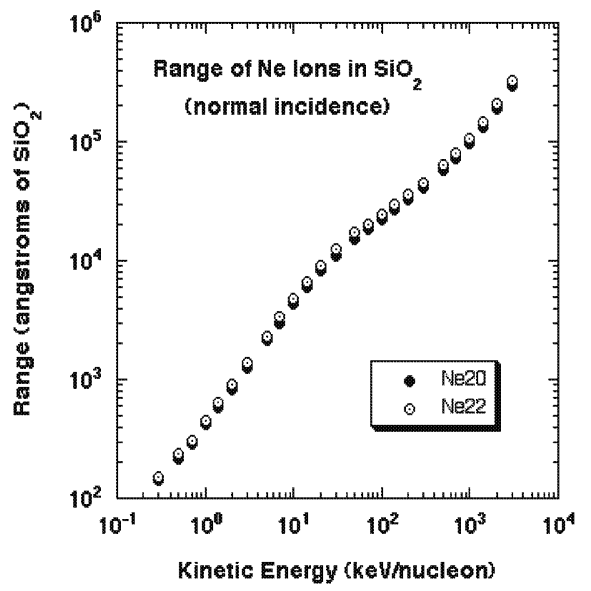

Figure 3: Range of ${ }^{20} \mathrm{Ne}$ and ${ }^{22} \mathrm{Ne}$ in $\mathrm{SiO}_{2}$ at normal incidence calculated by the SRIM 2000 version of TRIM.

In order to simulate the spectrum and behavior of interplanetary ions the oxygen spectrum in Figure 1 was divided into ten energy-bins per decade with a logarithmic spacing factor of $10^{0.1}=1.256$. For example, ions with energies from $E_{1} \mathrm{keV} /$ nucleon to $E_{2}$ $=1.256 \mathrm{E}_{1} \mathrm{keV} /$ nucleon were represented by a single energy $\langle\mathrm{E}\rangle=\left(\mathrm{E}_{1} \mathrm{E}_{2}\right)^{1 / 2} \mathrm{keV} /$ nucleon. A total of 42 bins were used to represent the observed spectrum from $\sim 0.2$ $\mathrm{keV} /$ nucleon to $3 \mathrm{MeV} /$ nucleon.

The solar wind (which travels more or less as a beam) can only impact the Sun-facing side of the Moon, while particles with several times solar wind speed are assumed to have an isotropic distribution with access to all sides of the Moon. The solar wind (SW) and suprathermal tail distributions were therefore treated separately to allow for their different patterns of incidence and possibly different isotopic composition. Within each energy bin from 0.2 to $12 \mathrm{keV} /$ nucleon, Monte Carlo trajectories were calculated with TRIM for ${ }^{20} \mathrm{Ne}$ and ${ }^{22} \mathrm{Ne}$ ions (a total of 2000 ions for each energy) distributed in eight angular intervals.

The distribution of incidence angles for the solar wind reflects the changing angle of incidence during the Moon's orbit about the Earth. The spectrum of energetic particles from $5 \mathrm{keV} /$ nucleon to $3 \mathrm{MeV} /$ nucleon (the suprathermal, or SEP component) was assumed to have a power-law spectrum with a slope of -2 . Trajectories were calculated for $2000{ }^{20} \mathrm{Ne}$ and ${ }^{22} \mathrm{Ne}$ ions in each energy bin, in this case distributed between 16 angles to represent an isotropic flux. The depth distributions of ${ }^{20} \mathrm{Ne}$ and ${ }^{22} \mathrm{Ne}$ were accumulated in an array of bins from 1 angstrom to $3 \times 10^{5}$ angstroms, again with 10 logarithmically-spaced bins per decade (see Figure 4).

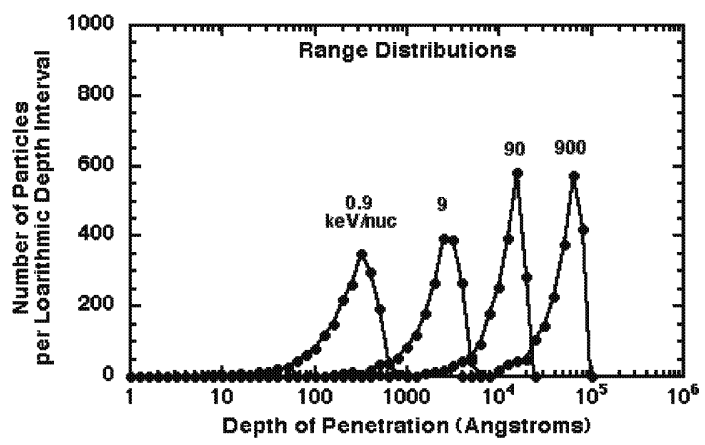

Figure 4: Depth distributions of ${ }^{20} \mathrm{Ne}$ isotopes at four energies (in $\mathrm{keV} /$ nucleon), taking into account the angular distributions of the incident ions (see text).

The information from these 96 runs can be combined to simulate arbitrary energy spectra and neon isotope compositions by adjusting the weighting of the various runs. For this study we assumed that the beam was pure solar wind for energies $<5 \mathrm{keV} /$ nucleon and pure suprathermal component for $>12 \mathrm{keV} /$ nucleon with a smoothly evolving mix at intermediate energies. The present-day solar-wind abundance ratio of ${ }^{20} \mathrm{Ne} /{ }^{22} \mathrm{Ne}=$ 13.7 [21] was assumed, independent of energy. The integrated interplanetary SEP/SW ratio is $\sim 3 \times 10^{-5}$ (see Figure 2).

Assuming SEP ions can access the lunar surface facing away from the Sun, the SEP/SW ratio in the implanted fluxes is $\sim 10^{-4}$. Figure 5 shows the depth distributions that result, including the integral distributions. The resulting ${ }^{20} \mathrm{Ne} /{ }^{22} \mathrm{Ne}$ ratio is shown in Figure 6, while Figure 7 shows ratios integrated over depth. We have not included the "cosmogenic" component, which includes roughly equal amounts of the three neon isotopes. This component, which results from cosmic-ray nuclear reactions with lunar material, is distributed uniformly throughout the samples.

Several features in the simulations are related to the input spectrum and the range-energy relation. Over a restricted interval the range-energy relation for $\mathrm{Ne}$ isotopes can be represented by $\mathrm{R}(\mathrm{E}, \mathrm{M})=\mathrm{a}(\mathrm{M} / 20) \mathrm{E}^{\mathrm{b}}$, where $a$ is a constant, $M$ is the mass in amu, and $b \approx 1$ for $0.5 \leq \mathrm{E} \leq 30 \mathrm{keV} /$ nucleon. Assuming a power-law spectrum $\left(\mathrm{dJ} / \mathrm{dE}=\mathrm{kE}^{\mathrm{s}}\right)$ with index $\mathrm{s}$, it can be shown that the relative abundance of stopping ${ }^{20} \mathrm{Ne}$ and ${ }^{22} \mathrm{Ne}$, $\left({ }^{20} \mathrm{Ne} /{ }^{22} \mathrm{Ne}\right)_{\mathrm{d}}$, is related to the interplanetary ratio, $\left({ }^{20} \mathrm{Ne} /{ }^{22} \mathrm{Ne}\right)_{\mathrm{i}}$ by

$$
\left({ }^{20} \mathrm{Ne} /{ }^{22} \mathrm{Ne}\right)_{\mathrm{d}}=(22 / 20)^{(\mathrm{s}+1) / \mathrm{b}}\left({ }^{20} \mathrm{Ne} /{ }^{22} \mathrm{Ne}\right)_{\mathrm{i}} .
$$




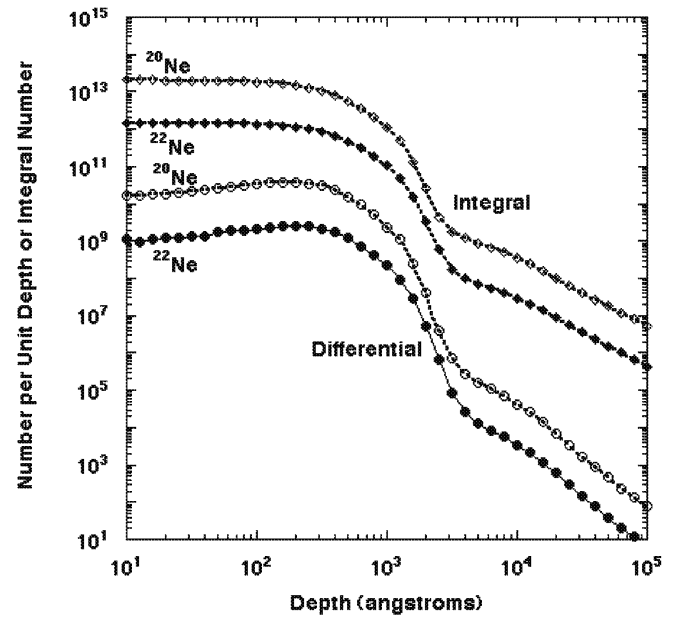

Figure 5: Simulated depth distributions of ${ }^{22} \mathrm{Ne}$ and ${ }^{20} \mathrm{Ne}$ based on the observed oxygen energy spectrum.

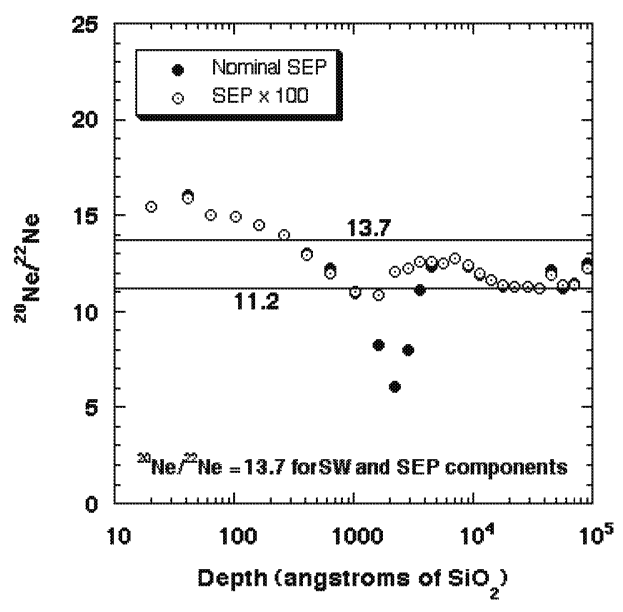

Figure 6: Ratio of ${ }^{20} \mathrm{Ne} /{ }^{22} \mathrm{Ne}$ vs. depth for spectra in Figure 5 (solid points). For the open points the SEP (or suprathermal component was multiplied by x100. Below 2000 angstroms two adjacent bins were combined. Outside the 1000-5000 angstrom range, the open and closed points are essentially identical. The SW and SEP values are indicated.

The mean range of $\sim 1 \mathrm{keV} /$ nucleon solar wind is $\sim 400$ angstroms in $\mathrm{SiO}_{2}$. At depths $<400$ angstroms the ${ }^{20} \mathrm{Ne} /{ }^{22} \mathrm{Ne}$ abundance ratio in the implanted ions is greater than the solar wind ratio of 13.7 because ${ }^{22} \mathrm{Ne}$ penetrates somewhat deeper on average. Thus, for $b \approx$ 1 and $\mathrm{s}>0$ we expect $\left({ }^{22} \mathrm{Ne} /{ }^{20} \mathrm{Ne}\right)_{\mathrm{d}}>13.6$ from equation (1). For depths from $\sim 400$ to $\sim 3000$ angstroms the solar wind spectral slope becomes more and more negative and the ${ }^{20} \mathrm{Ne} /{ }^{22} \mathrm{Ne}$ ratio at a given depth decreases, reaching a minimum at $\sim 3000$ angstroms at a point corresponding to the steepest part of the solar wind energy spectrum.
Particles at depths from 4000 to $\sim 12000$ angstroms are derived from the suprathermal spectrum from $\sim 8$ to $\sim 25 \mathrm{keV} /$ nucleon, and we expect $\left({ }^{20} \mathrm{Ne} /{ }^{22} \mathrm{Ne}\right)_{\mathrm{d}} \approx 12.2$ for $\mathrm{s}=-2$ and $\mathrm{b}=1$. From $\sim 30$ to $\sim 300 \mathrm{keV} /$ nucleon the range-energy relation flattens to $\mathrm{b} \approx 0.5$ to 0.6 (see Figure 3), and we expect $\left({ }^{20} \mathrm{Ne} /{ }^{22} \mathrm{Ne}\right)_{\mathrm{d}} \approx 11.3$ for $\mathrm{s}=-2$ from $\sim 15000$ to 50000 angstroms. Beyond $\sim 300$ $\mathrm{keV} /$ nucleon, the range-energy slope $\mathrm{b}$ again increases, causing the ratio to increase somewhat. The behavior predicted by the simulations differs somewhat from the predictions of Eq. (1) because of the range of angles of incidence and limitations of the power-law assumptions for $\mathrm{b}$ and $\mathrm{s}$. There are also minor fluctuations that may result from beating between the energy and depth bins. However, we conclude that the spectral slope and range-energy relation govern the concentration of $\mathrm{Ne}$ isotopes implanted at a given depth.

\section{DISCUSSION}

The simulations in Figures 6 and 7 illustrate that even with a constant isotope ratio at all energies, one expects significant variations in ${ }^{20} \mathrm{Ne} /{ }^{22} \mathrm{Ne}$ as a function of depth as a result of changes in slope of the input spectrum and the range-energy relation. At a given depth the simulated ${ }^{20} \mathrm{Ne} /{ }^{22} \mathrm{Ne}$ ratio (Figure 6) ranges from $\sim 16$ down to $\sim 6$, and the interplanetary abundance ratio of $\sim 13.7$ is achieved only briefly. It is not clear to what extent these predicted features are reflected in the lunar sample data. In typical 3-isotope plots of stepwise etching data [7] the measured ratios range from $\sim 13$ to 14 down to $\sim 11$ for very low ${ }^{21} \mathrm{Ne}$ abundance. At greater depths, where cosmogenic neon dominates, the ${ }^{20} \mathrm{Ne} /{ }^{22} \mathrm{Ne}$ and ${ }^{21} \mathrm{Ne} /{ }^{22} \mathrm{Ne}$ ratios gradually approach $\sim 1$.

There are several reasons why the simulations in Figures 6 and 7 might not correspond directly with real measurements: (a) it is unlikely that samples have a uniformly flat surface oriented normal to the Sun and they have probably experienced several orientations; (b) there could be diffusion of $\mathrm{Ne}$ in the samples; (c) it appears that step-wise etching experiments release gas from a broad depth range ( $>1000$ angstroms) at a time, and (d) the etch rate is not uniform over the surface (R. Wieler, personal communication). All of these effects tend to smooth out the measured gas-release pattern.

The plot in Figure 6, with depth on a log scale, emphasizes variations at small depths. If imagined on a linear scale, which corresponds more with the gas release process, it is more apparent that the expected ${ }^{20} \mathrm{Ne} /{ }^{22} \mathrm{Ne}$ ratio is similar to that of the SEP component over a large depth interval, from $\sim 10^{4}$ to $\sim 10^{5}$ angstroms ( 1 to 10 microns; see also Figure 7 ). The SEP component has been identified at depths up to $\sim 35$ microns in the lunar soil [7]. 


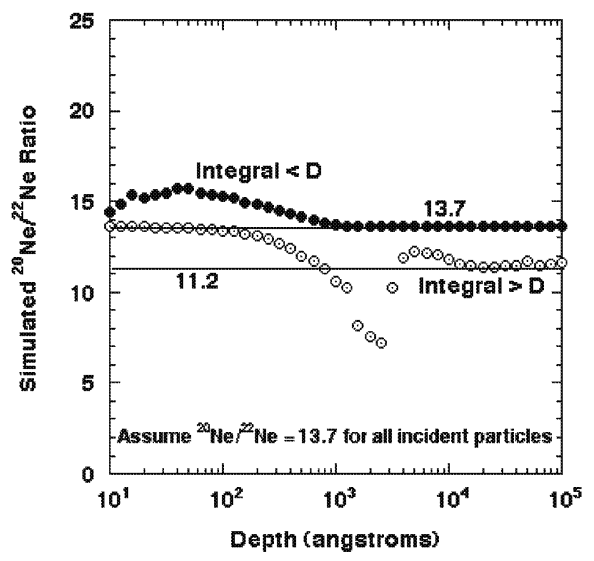

Figure 7: Ratios of ${ }^{20} \mathrm{Ne} /{ }^{22} \mathrm{Ne}$ in the integrated concentrations of Ne at depths $\angle \mathrm{D}$ and $>\mathrm{D}$. The SW and SEP values are indicated.

If no material is lost from a sample and the first etch step removes at least 1000 angstroms of material, a ratio of 13.6 is expected (the small difference with the assumed SW value of 13.7 is because TRIM finds that slightly more ${ }^{20} \mathrm{Ne}$ than ${ }^{22} \mathrm{Ne}$ is backscattered from the lunar surface without being implanted). Beyond the peak in the solar wind spectrum ( 400 angstroms) the expected ratio falls below 12 when $\sim 10 \%$ of the implanted gas remains. At depths greater than a few thousand angstroms the ratios in the simulation approach the measured SEP ratio of $\sim 11.2$, but by this point only $\sim 10^{-5}$ of the implanted gas remains. This latter value is far below the estimate that SEP neon represents $\sim 10 \%$ of the implanted $\mathrm{Ne}$.

There are several possible ways to increase the SEP abundance in lunar samples. Wieler [2] points out that only $\sim 10 \%$ of solar-wind Ne may be retained on impact. In addition, there may be weathering effects on the lunar surface that continually remove the outer layers of a grain. These two effects might increase the SEP/SW ratio by factor of $\sim 10$ to 100 [2]. There is support for these possibilities in the fact that none of the measurements in any of the five samples in Wieler et al. [7] give values as large as that in the contemporary solar wind $(=13.7)$, suggesting that ${ }^{20} \mathrm{Ne}$-rich material near the grain surface has been lost, and that the gas released in these measurements was implanted at a depth beyond 500 angstroms (see Figure 6). In contrast, studies of ilmenite samples (which retain noble gases more effectively), do measure the solar wind composition in the outermost grain layers [22]. Becker and Wieler (see [2]) have conducted simulations that include surface sputtering which indicate that the true SW ratio is obtained at the grain surface once a steady state is achieved.
The depth resolution of the lunar measurements should also be considered. Our simulation (Figure 6) gives a ${ }^{20} \mathrm{Ne} /{ }^{22} \mathrm{Ne}$ ratio of $<12.5$ for all depths $>600$ angstroms, corresponding to $\sim 20 \%$ of the total implanted neon. If the etch steps typically integrate over several thousand angstroms at a time and there is a substantial variation in the depth to which a given etch step penetrates, then it is possible to achieve ${ }^{20} \mathrm{Ne} /{ }^{22} \mathrm{Ne}$ abundance ratios significantly less that the solar wind value in $\sim 10 \%$ of the released gas without increasing the intensity of the SEP component. However, it is not clear that this possibility would explain the many lunarsample measurements to date.

It is interesting that none of the measurements that we are aware of find evidence for the region from $\sim 1000$ to 3000 angstroms where ${ }^{20} \mathrm{Ne} /{ }^{22} \mathrm{Ne} \approx 6-10$ (Figures 6 and 7). While the expected dip in the ${ }^{22} \mathrm{Ne} /{ }^{20} \mathrm{Ne}$ ratio at $1000-3000$ angstroms might be smoothed out by uneven etch steps that sample a range of depths and by weathering effects, as discussed above, it seems unlikely that this would explain the lack of evidence for this dip in the many samples studied.

Our simulations show that it is possible to mask the effects of the steep negative slope of the SW spectrum by increasing the intensity of the SEP component by a factor of $\sim 100$ (see Figure 6). Thus, the lack of evidence for measurements with ${ }^{20} \mathrm{Ne} /{ }^{22} \mathrm{Ne}=6$ to 10 supports the possibility that the SEP/SW ratio was much greater when these samples were exposed. A somewhat smaller increase in SEP/SW might be adequate (and/or the expected ${ }^{20} \mathrm{Ne} /{ }^{22} \mathrm{Ne}$ ratio $>600$ angstroms further smoothed) if weathering (sputtering) effects are included. Further work is needed to see if these combined effects can explain the available data.

It is possible that the fluence of suprathermal ions was greater in the past than it is today. This might be due to a general increase in solar activity, or there could be other causes. If the suprathermal tail on the solar wind is due to statistical-acceleration by interplanetary turbulence [15], an increase in the level of turbulence would presumably lead to a corresponding increase in the interplanetary fluxes. Or if most of the particles $>10$ $\mathrm{keV} /$ nucleon are CIR-accelerated particles, an increase in the frequency or speed of high-speed streams would cause increased interplanetary particle intensities.

While it is difficult to explain an SEP/SW ratio of 0.1 or more, as inferred from lunar samples, our simulation results show that it is possible to mimic a separate, more penetrating, isotopically-heavy component with a combination of energy-spectrum and range-energy effects. Although some of the effects of the difference in range between ${ }^{22} \mathrm{Ne}$ and ${ }^{20} \mathrm{Ne}$ have been recognized previously, the possibility that they might help explain the SEP component has generally 
been discounted [9]. Our results show that reduced ${ }^{20} \mathrm{Ne} /{ }^{22} \mathrm{Ne}$ ratios arise quite naturally over an extended range of depths. For example, for 30 to $300 \mathrm{keV} / \mathrm{nuc}$ ions, Eq. (1) with $\mathrm{b} \approx 0.5$ gives an $\mathrm{SEP}{ }^{20} \mathrm{Ne} /{ }^{22} \mathrm{Ne}$ ratio of 11.3 with the following combinations of source ratio and spectral index: ${ }^{20} \mathrm{Ne} /{ }^{22} \mathrm{Ne} \approx 11.3$ with $\mathrm{dJ} / \mathrm{dE} \sim$ $\mathrm{E}^{-1},{ }^{20} \mathrm{Ne} /{ }^{22} \mathrm{Ne} \approx 13.7$ with $\mathrm{dJ} / \mathrm{dE} \sim \mathrm{E}^{-2}$, or ${ }^{20} \mathrm{Ne} /{ }^{22} \mathrm{Ne} \approx$ 16.5 with $\mathrm{dJ} / \mathrm{dE} \sim \mathrm{E}^{-3}$. While any of these is possible, Occam's Razor suggests assuming the SW ratio of 13.7 unless there is good reason not to. Changing the suprathermal isotope ratio might achieve a ratio close to the SEP ratio over a broader depth range, but this is outside the scope of this paper.The spectral and rangeenergy effects discussed here will also produce correlated enhancements of the heavy isotopes of $\mathrm{He}$, $\mathrm{Ar}, \mathrm{Kr}$, and $\mathrm{Xe}$, as observed in lunar and meteoritic samples [2], and they can potentially also cause apparent enhancements of $\mathrm{Kr}$ and $\mathrm{Xe}$ with respect to lighter noble gases.

\section{SUMMARY}

In this paper we have made an initial attempt to simulate the depth distribution of $\mathrm{Ne}$ isotopes implanted in lunar samples using available rangeenergy routines and newly-measured spectra of solar wind and heliospheric particles. We find that the falling energy spectra, combined with the longer range of ${ }^{22} \mathrm{Ne}$, lead to an apparent enrichment in ${ }^{22} \mathrm{Ne}$ with respect to ${ }^{20} \mathrm{Ne}$ for all depths (in $\mathrm{SiO}_{2}$ ) beyond $\sim 400$ angstroms. In particular, at depths of $\sim 10^{4}$ to $10^{5}$ angstroms a ${ }^{22} \mathrm{Ne} /{ }^{20} \mathrm{Ne}=13.7$ source ratio transforms to 11.3 , similar to the SEP component in lunar samples. The mechanism also causes apparent enrichments of heavy $\mathrm{He}, \mathrm{Ar}, \mathrm{Kr}$, and Xe isotopes.

Although it is not yet clear to what extent these simulations are consistent with existing lunar sample data, these first results emphasize that interpretations of the composition of implanted ions require knowledge of their interplanetary energy spectrum. As for why the SEP component apparently comprises $\sim 10-40 \%$ of all implanted ions, we find that the expected ${ }^{22} \mathrm{Ne} /{ }^{20} \mathrm{Ne}$ ratio at depths beyond $\sim 600$ angstroms ( $20 \%$ of all implanted ions) is closer to the SEP value than to the (input) SW value. Improved agreement with the lunar data is achieved if the flux of suprathermal ions was $~ 100$ times greater in the past. Further work is needed to explore the degree to which present-day solar wind and suprathermal spectra can help interpret available lunar data.

Acknowledgments: This work was supported by NASA at Caltech (under grant NAS5-6912) and at the University of Maryland. We thank D. Burnett and M. Wiedenbeck for help with TRIM simulations and R,
Wimmer-Schweingruber for discussions of SEP neon. We greatly appreciate the assistance of R. Wieler in interpreting noble gas measurements in lunar samples.

\section{References}

1. Pepin, R. O. et al., Proc. Apollo 11 Lunar Science Conference, 1435, 1970.

2. Wieler, R., Space. Science Reviews 85, 303, 1998.

3. Kerridge, J. F., Rev. Geophys. 31, 423, 1993.

4. Black, D. C.and Pepin, R. O., Earth Planet Sci. Lett. 6, 395-405, 1969.

5. Black, D. C., Geochim. Cosmochim. Acta 36, 347-375, 1972.

6. Black, D. C., Ap. J., 266, 889, 1983,

7. Wieler, R., Baur, H., and Signer, P, Geochim. Cosmochim. Acta, 50, 1997-2017, 1986.

8. Geiss, J., and Bochsler, P., in The Sun in Time, C. P. Sonnett et al., eds, Univ. Arizona Press, Tucson 1991.

9. Wieler, R., Lunar and Planetary Science XXCVII, Lunar and Planetary Institute, Houston, p. 1551, 1997.

9. Wimmer-Schweingruber, R. F., Lunar Soils: A Long-Term Archive for the Galactic Environmant of the Solar System?, Habilitation Thesis University of Bern, 2000.

11. Wimmer-Schweingruber, R. F., and P. Bochsler, in Acceleration and Transport of Energetic Particles Observed in the Heliosphere (Mewaldt et al., eds.), AIP \#528, p. 270, 2000.

12. Wimmer-Schweingruber, $\mathrm{R}$, submitted to SOHO-ACE Workshop Proc. (AIP), 2001.

13. Mewaldt, R. A., et al., submitted to SOHO-ACE Workshop Proc. (AIP), 2001.

14. Gloeckler, G., Fisk, L. A., Zurbuchen, T. H., and Schwadron, N. A., in AIP Conf. Proc. 528, Acceleration and Transport of Energetic Particles Observed in the Heliosphere, ed. R. A. Mewaldt et al., (New York: AIP), 221, 2000.

15. Fisk, L. A., Gloeckler, G., Zurbuchen, T. H., and Schwadron, N. A., in AIP Conf. Proc. 528, Acceleration and Transport of Energetic Particles Observed in the Heliosphere, ed. R. A. Mewaldt et al., (New York: AIP), $229,2000$.

16. Leske, R. A. et al., submitted to SOHO-ACE Workshop Proc. (AIP), 2001.

17. Mason et al. Ap.J. 425, 843, 1994.

18. Dwyer, J. R., et al., Proc. $26^{\text {th }}$ Internat. Cosmic Ray Conf. (Salt Lake City), 6, 147, 1999.

19, Slocum, P. S., et al., submitted to SOHO-ACE Workshop Proc. (AIP), 2001.

20. Ziegler, J. F., Biersack, J. P., and Littmark, U., The Stopping and Range of Ions in Solids, Vol. 1., Pergammon Press, New York, New York, 1985.

21. Geiss, J., et al. in Apollo-16 Preliminary Science Report, NASA SP-315, 231, p. 14-1, 1972.

22. Benkert J.P, Baur, H., Signer, P., and Wieler, R., JGR 98, 13147-13162, 1993. 